\title{
UNITARITY AND REAL PROPERTIES OF THE NEUTRAL MESON COMPLEX
}

\author{
K. Urbanowski, J. Jankiewicz \\ University of Zielona Góra, Institute of Physics, \\ ul. Prof. Z. Szafrana 4a, 65-516 Zielona Góra, Poland
}

(Received September 06, 2010; received in final form February 06, 2011)

\begin{abstract}
The proof of the Khalfin Theorem for the neutral meson complex is analyzed. It is shown that the unitarity of the time evolution operator for the total system under considerations assures that the Khalfin's Theorem holds. The consequences of this Theorem for the neutral mesons system are discussed: it is shown that diagonal matrix elements of the exact effective Hamiltonian for the neutral meson complex cannot be equal if CPT symmetry holds and CP symmetry is violated. The properties of time evolution governed by a time-independent effective Hamiltonian acting in the neutral mesons subspace of states are considered. Using the Khalfin's Theorem it is shown that if such Hamiltonian is time-independent then the evolution operator for the total a system containing the neutral meson complex cannot be a unitary operator. It is shown graphically for a given specific model how the Khalfin's Theorem works. It is also shown for this model how the difference of the mentioned diagonal matrix elements of the effective Hamiltonian varies in time.
\end{abstract}

Key words: CP violation, CPT symmetry, neutral mesons.

PACS number(s): 03.65.Ca, 11.30.Er, 11.10.St, 14.40.Aq

\section{INTRODUCTION}

The standard method used for the description of the properties of two particle (two state) complexes is the Lee-Oehme-Yang (LOY) approximation [1-7]. This approximation was applied by LOY to the description and analysis of the decay of neutral kaons. Its source is the well known Weisskopf-Wigner (WW) theory of the decay processes [8]. Within this approach the solutions of the Schödinger equation

$$
i \frac{\partial|\psi ; t\rangle}{\partial t}=H|\psi ; t\rangle, \quad|\psi ; t=0\rangle=\left|\psi_{0}\right\rangle,
$$

(where $H$ is the total selfadjoint Hamiltonian for the system containing neutral kaons and units $\hbar=c=1$ are used) describe time evolution of vectors $|\psi ; t\rangle$ in the Hilbert space, $\mathcal{H}$, of states of the total system under considerations and the Hamiltonian $H$ for the problem is divided into two parts $H^{(0)}$ and $H^{(1)}$ :

$$
H=H^{(0)}+H^{(1)}
$$

such that $\left|K_{0}\right\rangle \equiv|\mathbf{1}\rangle$ and $\left|\bar{K}_{0}\right\rangle \equiv|\mathbf{2}\rangle$ are discrete eigenstates of $H^{(0)}$ for the 2-fold degenerate eigenvalue $m_{0}$,

$$
H^{(0)}|\mathbf{j}\rangle=m_{0}|\mathbf{j}\rangle, \quad(j=1,2) ;
$$

where $\langle\mathbf{j} \mid \mathbf{k}\rangle=\delta_{j k}$ and $H^{(1)}$ induces the transitions from eigenstates $|\mathbf{j}\rangle$ to other (unbound) eigenstates $|\varepsilon, J\rangle$ of $H^{(0)}$,

$$
H^{(0)}|\varepsilon, J\rangle=\varepsilon|\varepsilon, J\rangle,
$$

and, consequently, also between $\left|K_{0}\right\rangle$ and $\left|\bar{K}_{0}\right\rangle$. (We have $\left\langle\varepsilon^{\prime}, J_{1} \mid \varepsilon, J_{2}\right\rangle=\delta_{J_{1} J_{2}} \delta\left(\varepsilon-\varepsilon^{\prime}\right),\langle\varepsilon, J \mid \mathbf{k}\rangle=0, j, k=1,2$, and $J$ denotes such quantum numbers as charge, spin, etc.). So, the problem which one usually considers is the time evolution of an initial state, which is a superposition of $|\mathbf{1}\rangle$ and $|\mathbf{2}\rangle$ states [1].
In the kaon rest-frame, this time evolution for $t \geq t_{0} \equiv$ 0 is governed by the Schrödinger equation (1), whose solutions $|\psi ; t\rangle$ have the following form $[1,4,5]$

$$
|\psi ; t\rangle=a_{1}(t)|\mathbf{1}\rangle+a_{2}(t)|\mathbf{2}\rangle+\sum_{J, \varepsilon} F_{J}(\varepsilon ; t)|\varepsilon, J\rangle,
$$

where

$$
\begin{gathered}
\left|a_{1}(t)\right|^{2}+\left|a_{2}(t)\right|^{2}+\sum_{J, \varepsilon}\left|F_{J}(\varepsilon, t)\right|^{2}=1, \\
F_{J}(\varepsilon ; t=0)=0 .
\end{gathered}
$$

Here $\sum_{\varepsilon} F_{J}(\varepsilon ; t)|\varepsilon, J\rangle$ represents the decay products in the channel $J$.

Inserting (4) into the Schrödinger equation (1) leads to a system of coupled equations for amplitudes $a_{1}(t)$, $a_{2}(t)$ and $F_{J}(\varepsilon ; t)$. Adopting the WW approximations to these equations and solving them LOY obtained their approximate equations for $a_{1}(t), a_{2}(t)[1,4,9]$. From this we get:

$$
i \frac{\partial}{\partial t}|\psi ; t\rangle_{\|}=H_{\mathrm{LOY}}|\psi ; t\rangle_{\|}, \quad\left(t \geq t_{0}\right)
$$

where

$$
|\psi ; t\rangle_{\|}=a_{1}(t)|\mathbf{1}\rangle+a_{2}(t)|\mathbf{2}\rangle
$$

and

$$
H_{\mathrm{LOY}} \equiv M_{\mathrm{LOY}}-\frac{i}{2} \Gamma_{\mathrm{LOY}}
$$

Here $M_{\mathrm{LOY}}=M_{\mathrm{LOY}}^{+}, \Gamma_{\mathrm{LOY}}=\Gamma_{\mathrm{LOY}}^{+} \cdot H_{\mathrm{LOY}}$ is $(2 \times 2)$ matrix acting in a two-dimensional subspace (let us denote it by $\left.\mathcal{H}_{\|}\right)$of $\mathcal{H}$ spanned by vectors $|\mathbf{1}\rangle,|\mathbf{2}\rangle$. Thus $|\psi ; t\rangle_{\|} \in$ $\mathcal{H}_{\|}$and $h_{j k}^{\mathrm{LOY}}=\left\langle\mathbf{j}\left|H_{\mathrm{LOY}}\right| \mathbf{k}\right\rangle, M_{j k}^{\mathrm{LOY}}=\left\langle\mathbf{j}\left|M_{\mathrm{LOY}}\right| \mathbf{k}\right\rangle$, $\Gamma_{j k}^{\mathrm{LOY}}=\left\langle\mathbf{j}\left|\Gamma_{\mathrm{LOY}}\right| \mathbf{k}\right\rangle$. 


\section{K. URBANOWSKI, J. JANKIEWICZ}

The eigenvectors, $\left|K_{S}\right\rangle,\left|K_{L}\right\rangle$, for $H_{\mathrm{LOY}}$ to the eigenvalues, $\mu_{S}=m_{S}-\frac{i}{2} \gamma_{S}$ and $\mu_{L}=m_{L}-\frac{i}{2} \gamma_{L}$, have the following form

$$
\begin{aligned}
& \left|K_{S}\right\rangle=p_{S}\left|K_{0}\right\rangle-q_{S}\left|\bar{K}_{0}\right\rangle, \\
& \left|K_{L}\right\rangle=p_{L}\left|K_{0}\right\rangle+q_{L}\left|\bar{K}_{0}\right\rangle,
\end{aligned}
$$

where,

$$
\left|p_{S}\right|^{2}+\left|q_{S}\right|^{2}=\left|p_{L}\right|^{2}+\left|q_{L}\right|^{2}=1
$$

Now, if one assumes that the total system under considerations is CPT-invariant,

$$
[\Theta, H]=0,
$$

where $\Theta$ is an antiunitary operator:

$$
\Theta \stackrel{\text { def }}{=} \mathcal{C} \mathcal{P} \mathcal{T}
$$

and $\mathcal{C}$ is the charge conjugation operator, $\mathcal{P}$ is space inversion, and the antiunitary operator $\mathcal{T}$ represents the time reversal operation, one easily finds using explicit formulae for $h_{j k}^{\mathrm{LOY}}[1-7]$, [10] that in such a case the diagonal matrix elements of $H_{\mathrm{LOY}}$ must be equal:

$$
h_{11}^{\mathrm{LOY}}=h_{22}^{\mathrm{LOY}} .
$$

One of the consequences of property (13) is that in the CPT invariant systems

$$
p_{S}=p_{L} \equiv p, \quad q_{S}=q_{L} \equiv q,
$$

in (9), (10) and

$$
\left(\frac{q}{p}\right)^{2}=\frac{h_{21}^{\mathrm{LOY}}}{h_{12}^{\mathrm{LOY}}}=\text { const. }
$$

Within this approach there is $\left|\frac{q}{p}\right| \neq 1$ in CPT invariant system when CP is violated [10]. This property and properties (13), (14) are the standard result of the LOY approach and this is the picture which one meets in the literature [1-7].

Note that if one describes the properties of neutral mesons and the time evolution of their state vectors using the LOY method then, in fact, one assumes that the selfadjoint Hamiltonians $H, H^{(0)}$ and $H^{(1)}$ acting in $\mathcal{H}$ exist and that the solutions of Schrödinger equation (1) describe the time evolution of states in $\mathcal{H}$. There is no LOY method and no LOY approximation without these Hamiltonians and without the Schrödinger equation.

The aim of this paper is to confront the main predictions of the LOY theory such as (13), (14), etc., with the predictions following from the rigorous treatment of two state quantum mechanical subsystems and from the properties of the exact effective Hamiltonian for such subsystems. Sec. II contains the proof of the Khalfin's Theorem [11-18]. In Sec. III the properties of the time evolution governed by a time independent effective Hamiltonian acting in a two-dimensional subspace and of the evolution operator for this case are analyzed and confronted with the conclusions following from the Khalfin's Theorem. In Sec. IV the properties of the exact effective Hamiltonian for two-state subsystems and consequences of the above-mentioned Theorem are discussed. In Sec. V using a model of neutral kaon complex the results of calculations showing how the Khalfin's Theorem "works" are presented graphically. Section VI contains some final remarks.

\section{KHALFIN'S THEOREM}

From the general principles of quantum mechanics it follows that transitions of the system from a state $\left|\psi_{1}\right\rangle \in \mathcal{H}$ at the time $t=0$ to the state $\left|\psi_{2}\right\rangle \in \mathcal{H}$ at the time $t>0,\left|\psi_{1}\right\rangle \stackrel{t}{\rightarrow}\left|\psi_{2}\right\rangle$, are governed by Eq (1) with a time independent Hamiltonian and realized by the transition (evolution) unitary operator $U(t)$ acting in $\mathcal{H}$, such that

$$
U\left(t_{1}\right) U\left(t_{2}\right)=U\left(t_{1}+t_{2}\right)=U\left(t_{2}\right) U\left(t_{1}\right) .
$$

From this condition and from the unitarity it follows that

$$
U(0)=\mathbb{I} \text { and }[U(t)]^{-1} \equiv[U(t)]^{+}=U(-t),
$$

where $\mathbb{I}$ is the unit operator in $\mathcal{H}$.

The probability to find the system in the state $\left|\psi_{j}\right\rangle$ at the time $t$ if it were earlier at an instant $t=0$ in the initial state $\left|\psi_{k}\right\rangle$ is determined by the transition amplitude $A_{j k}(t)$,

$$
A_{j k}(t)=\left\langle\psi_{j}|U(t)| \psi_{k}\right\rangle
$$

where $(j, k=1,2)$. Using (16) and following [14] it can be easily found that

$$
\left[A_{12}(-t)\right]^{*}=A_{21}(t) .
$$

So, defining the function [14]

$$
f_{21}(t) \stackrel{\text { def }}{=} \frac{A_{21}(t)}{A_{12}(t)},
$$

and taking into account the general property (18) one finds that the function $f_{21}(t)$ must satisfy the relation

$$
\left[f_{21}(-t)\right]^{*} f_{21}(t)=1 \text {. }
$$

Note that this last relation as well as property (18) are valid for any two states $\left|\psi_{1}\right\rangle,\left|\psi_{2}\right\rangle \in \mathcal{H}$.

The Kalfin's Theorem concerns one of the basic properties of any two state subsystem and, in fact, it is not limited to only such subsystems as the neutral meson complexes. This Theorem states that [11-18],

\section{Khalfin's Theorem}

If

$$
f_{21}(t)=\rho=\text { const. }
$$

then there must be

$$
R=|\rho|=1 \text {. }
$$


Indeed, from (20) it follows that if $f_{21}(t)=\rho=$ const for every $t \geq 0$ then $\left[f_{21}\left(t^{\prime}\right)\right]^{*}=\zeta=$ const for all $t^{\prime} \leq 0$. Now, if the functions $f_{21}(t)$ and $\left[f_{21}\left(t^{\prime}\right)\right]^{*}$ are continuous at $t=t^{\prime}=0$ then there must be

$$
R=|\rho|=|\zeta|=1,
$$

which is the proof of the Khalfin's Theorem.

The only problem in the above proof, as it was pointed out in [14], is to find conditions guaranteeing the continuity of $f_{21}(t)$ at $t=0$. There are two possibilities. The first: vectors $\left|\psi_{1}\right\rangle,\left|\psi_{2}\right\rangle$ are not orthogonal,

$$
\left\langle\psi_{1} \mid \psi_{2}\right\rangle \neq 0 \text {. }
$$

and the second one: these vectors are orthogonal

$$
\left\langle\psi_{j} \mid \psi_{k}\right\rangle=\delta_{j k}, \quad(j, k=1,2) .
$$

Case (23) is simple. One can always write

$$
\left|\psi_{2}\right\rangle=\left|\psi_{2}\right\rangle_{\|}+\left|\psi_{2}\right\rangle_{\perp},
$$

where

$$
\left\langle\psi_{1} \mid \psi_{2}\right\rangle_{\|} \neq 0, \quad \text { and } \quad\left\langle\psi_{1} \mid \psi_{2}\right\rangle_{\perp}=0 \text {, }
$$

In such a case from (16), (17) it follows that $A_{21}(0)=$ ${ }_{\|}\left\langle\psi_{2} \mid \psi_{1}\right\rangle=\left[\left\langle\psi_{1} \mid \psi_{2}\right\rangle_{\|}\right]^{*} \neq 0$ and thus $A_{12}(0) \equiv$ $\left[A_{21}(0)\right]^{*} \neq 0$ which yields

$$
\lim _{t \rightarrow 0+} f_{21}(t)=\frac{\left[\left\langle\psi_{1} \mid \psi_{2}\right\rangle_{\|}\right]^{*}}{\left\langle\psi_{1} \mid \psi_{2}\right\rangle_{\|}} \stackrel{\text { def }}{=} \rho_{1},
$$

where $\left|\rho_{1}\right|=1$, and

$$
\lim _{t^{\prime} \rightarrow 0-}\left[f_{21}\left(t^{\prime}\right)\right]^{*} \equiv \frac{1}{\rho_{1}}
$$

These last two relations mean that in the considered case (23) the functions $\left.f_{21}(t)\right|_{t \geq 0}$ as well as $\left.\left[f_{21}\left(t^{\prime}\right)\right]^{*}\right|_{t^{\prime} \leq 0}$ are continuous at $t=t^{\prime}=0$.

Now let us concentrate our attention on case (24). This situation occurs in the case of the neutral meson complexes but also it can be met in other cases. In general vectors $\left|\psi_{1}\right\rangle,\left|\psi_{2}\right\rangle$ need not describe the states of the neutral meson-antimeson pairs.

In the considered case (24), from (16), (17) and (24) one can see that $A_{21}(0)=0$ and $A_{12}(0)=0$ which by (19) means that without some additional conditions the function $f_{21}(t)$ need not be continuous at $t=0$. Taking into account that quantum theory requires $U(t)$ to have the form,

$$
U(t)=e^{-i t H},
$$

(using units $\hbar=1$ ), where $H$ is the total hermitian Hamiltonian of the system, (or, in the interaction picture

$$
U_{I}(t)=\mathbb{T} e^{-i \int_{0}^{t} H_{I}(\tau) d \tau}
$$

where $\mathbb{T}$ denotes the usual time ordering operator and $H_{I}(\tau)$ is the operator $H$ in the interaction picture), one can easily verify that to assure the continuity of $f_{21}(t)$ at $t=0$ it suffices that there exist such $n \geq 1$ that

$$
\begin{aligned}
& \left\langle\psi_{2}\left|H^{k}\right| \psi_{1}\right\rangle=0, \quad(0 \leq k<n), \\
& \left\langle\psi_{2}\left|H^{n}\right| \psi_{1}\right\rangle \neq 0 \quad \text { and } \quad\left|\left\langle\psi_{2}\left|H^{n}\right| \psi_{1}\right\rangle\right|<\infty .
\end{aligned}
$$

Assuming that this property holds and using the d'Hospital rule one finds that simply

$$
\lim _{t \rightarrow 0+} f_{21}(t)=\frac{\left\langle\psi_{2}\left|H^{n}\right| \psi_{1}\right\rangle}{\left\langle\psi_{1}\left|H^{n}\right| \psi_{2}\right\rangle},
$$

which means that $\left.f_{21}(t)\right|_{t \geq 0}$ is continuous at $t=0$. Similarly, the continuity of $\left.\left[f_{21}\left(t^{\prime}\right)\right]^{*}\right|_{t^{\prime} \leq 0}$ at $t^{\prime}=0$ is assured.

One of the aims of this paper is to consider the consequences of the Khalfin's Theorem for neutral meson complexes. In the case of neutral mesons $\psi_{1}=K_{0}, B_{0}, D_{0} \ldots$ and $\psi_{2}=\bar{K}_{0}, \bar{B}_{0}, \bar{D}_{0} \ldots$. Thus in a general case the subspace of states of neutral mesons, $\mathcal{H}_{\|}$, is a twodimensional subspace of $\mathcal{H}$ spanned by orthogonal vectors $\left|\psi_{1}\right\rangle,\left|\psi_{2}\right\rangle$. For neutral meson complexes according to the experimental results the particle-antiparticle transitions $\left|\psi_{1}\right\rangle \rightleftharpoons\left|\psi_{2}\right\rangle$ exist, which means that there must exist $n<\infty$ such that relation (31) occurs. It is known form the experiments that the transitions $|\Delta S|=2$ exist, so in this case $n \leq 2$. This means that in fact for the neutral meson complexes, where the transitions $\left|\psi_{1}\right\rangle \rightleftharpoons\left|\psi_{2}\right\rangle$ take place, only the assumption of unitarity of the exact transition operator $U(t)$ assures the validity of the Khalfin's Theorem and no more assumptions (eg. of the type that the CPT symmetry holds in the total system under considerations) are required.

The above considerations are completed in Sec. V, where it is shown in Fig. 1 how the Khalfin's Theorem "works" in a given model.

\section{TIME EVOLUTION IN $\mathcal{H}_{\|}$GOVERNED BY A TIME-INDEPENDENT HAMILTONIAN $H_{\|}$}

In this and subsequent Sections we will assume that the two-dimensional subspace $\mathcal{H}_{\|}$of $\mathcal{H}$ is spanned by orthogonal vectors $\left|\psi_{1}\right\rangle,\left|\psi_{2}\right\rangle,(24)$. So let us assume that the evolution operator $U_{\|}(t)$ acting in this $\mathcal{H}_{\|}$has the following form

$$
U_{\|}(t)=e^{-i t H_{\|}}
$$

and that the operator $H_{\|}$is a non-hermitian timeindependent $(2 \times 2)$ matrix acting in $\mathcal{H}_{\|}$,

$$
\frac{\partial h_{j k}}{\partial t}=0
$$

where $h_{j k}=\left\langle\psi_{j}\left|H_{\|}\right| \psi_{k}\right\rangle,(j, k=1,2)$. It is obvious that the operator $U_{\|}(t)$ is the $(2 \times 2)$ matrix and

$$
U_{\|}\left(t_{1}\right) U_{\|}\left(t_{2}\right)=U_{\|}\left(t_{2}\right) U_{\|}\left(t_{1}\right)=U_{\|}\left(t_{1}+t_{2}\right)
$$

and

$$
U_{\|}(0)=\mathbb{I}_{\|},
$$




\section{K. URBANOWSKI, J. JANKIEWICZ}

where $\mathbb{I}_{\|}$is the unit matrix in $\mathcal{H}_{\|}$.

It is easy to verify that the operator $U_{\|}(t)$ is the solution of the Schrödinger-like evolution equation for the subspace $\mathcal{H}_{\|}$,

$$
i \frac{\partial}{\partial t} U_{\|}(t)|\psi\rangle_{\|}=H_{\|} U_{\|}(t)|\psi\rangle_{\|}, \quad U_{\|}(0)=\mathbb{I}_{\|},
$$

where $|\psi\rangle_{\|} \in \mathcal{H}_{\|}$. Note that this last equation is the equation of the same type as the evolution equation used within the Lee-Oehme-Yang theory to describe the time evolution in neutral mesons subspace of states.

Using Pauli matrices $\sigma_{x}, \sigma_{y}, \sigma_{z}$ the matrix $H_{\|}$can be expressed as follows [19,20]

$$
H_{\|}=h_{0} \mathbb{I}_{\|}+\vec{h} \cdot \vec{\sigma},
$$

where

$$
\vec{h} \cdot \vec{\sigma}=h_{x} \sigma_{x}+h_{y} \sigma_{y}+h_{z} \sigma_{z},
$$

and $h_{0}=\frac{1}{2}\left(h_{11}+h_{22}\right), h_{z}=\frac{1}{2}\left(h_{11}-h_{22}\right)$, etc.

Within the use of the relation (37) the operator $U_{\|}(t)$ given by (33) can be rewritten in the following form

$$
\begin{aligned}
& U_{\|}(t)=e^{-i t H_{\|}} \equiv u_{0}(t) \mathbb{I}_{\|}+\vec{u}(t) \cdot \vec{\sigma}
\end{aligned}
$$

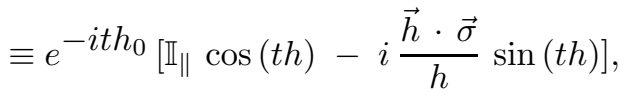

where

$$
\begin{aligned}
u_{0}(t) & =\frac{1}{2}\left(u_{11}(t)+u_{22}(t)\right), \\
u_{j k} & \stackrel{\text { def }}{=}\left\langle\psi_{j}\left|U_{\|}(t)\right| \psi_{k}\right\rangle, \quad(j, k=1,2), \\
\vec{u}(t) \cdot \vec{\sigma} & =u_{x}(t) \sigma_{x}+u_{y}(t) \sigma_{y}+u_{z}(t) \sigma_{z}, \\
h^{2} & =\vec{h} \cdot \vec{h}=h_{x}^{2}+h_{y}^{2}+h_{z}^{2} .
\end{aligned}
$$

Now taking into account that simply (see (37)),

$$
\vec{h} \cdot \vec{\sigma} \equiv H_{\|}-h_{0} \mathbb{I}_{\|},
$$

from (38) one finds

$$
\begin{aligned}
& u_{12}(t)=-i e^{-i t h_{0}} \frac{h_{12}}{h} \sin (t h), \\
& u_{21}(t)=-i e^{-i t h_{0}} \frac{h_{21}}{h} \sin (t h), \\
& u_{11}(t)=e^{-i t h_{0}}\left[\cos (t h)-i \frac{h_{z}}{h} \sin (t h)\right], \\
& u_{22}(t)=e^{-i t h_{0}}\left[\cos (t h)+i \frac{h_{z}}{h} \sin (t h)\right] .
\end{aligned}
$$

Relations (41) and (42) yield

$$
\frac{u_{21}(t)}{u_{12}(t)} \equiv \frac{h_{21}}{h_{12}} \stackrel{\text { def }}{=} r=\text { const. }
$$

Another useful relation resulting from (43) and (44) is the following one

$$
u_{11}(t)-u_{22}(t)=-2 i e^{-i t h_{0}} \frac{h_{z}}{h} \sin (t h) .
$$

So if one has any time-independent effective Hamiltonian $H_{\|}$acting in $\mathcal{H}_{\|}$and the evolution operator $U_{\|}(t)$ for $\mathcal{H}_{\|}$ has the form $U_{\|}(t)=e^{-i t H_{\|}}$then

$$
u_{11}(t)=u_{22}(t) \Leftrightarrow h_{11}=h_{22} .
$$

This property is quite independent of the relations of type (45).

All the above properties, including (45), (47), are true for every time-independent effective Hamiltonian $H_{\|}$acting in the two-dimensional subspace $\mathcal{H}_{\|}$. In other words, they hold for the LOY effective Hamiltonian, $H_{\mathrm{LOY}}$, as well as for every $H_{\|} \neq H_{\mathrm{LOY}}$.

The conclusion following from Khalfin's Theorem, (21), (22) and from (45) seems to be important,

\section{Conclusion 1}

If $|r| \neq 1$ and the time-independent effective Hamiltonian $H_{\|}$is the exact effective Hamiltonian for the subspace $\mathcal{H}_{\|}$of states of neutral mesons, that is if

$$
u_{j k}(t) \equiv A_{j k}(t)
$$

where $j \neq k,(j, k=1,2), r$ is defined by $(45)$ and $u_{j k}(t)$, $A_{j k}(t)$ are given by $(39)$ and (17) respectively, then the evolution operator $U(t)$ for the total state space $\mathcal{H}$ cannot be a unitary one.

Indeed, experimental results indicate that for the neutral kaon complex $|r| \neq 1$ (see, e. g. [10]). So, this conclusion holds because from the Khalfin's Theorem it follows that if $|r| \neq 1$ and matrix elements $A_{j k}(t), \quad(j, k=1,2)$ are the matrix elements of the exact evolution operator $U(t)$ then there must be $|r| \neq$ const. Thus if the relation (48) is the true relation then there is only one possibility: The Khalfin's Theorem is not applicable in this case. From the proof of this Theorem given in the previous Section and analysis of the case of neutral mesons performed there it follows that this Theorem holds if the evolution operator $U(t)$ for the total state space $\mathcal{H}$ of the system containing two state subsystem under consideration is a unitary operator. For the neutral mesons subsystem Khalfin's Theorem need not hold only if the total evolution operator $U(t)$ is not a unitary operator.

\section{SYMMETRIES CP, CPT AND THE EXACT EFFECTIVE HAMILTONIAN FOR THE NEUTRAL MESONS SUBSYSTEM}

The exact transition (evolution) operator for the subspace $\mathcal{H}_{\|}$can be found using the projection operator, $P$, defining this subspace, $\mathcal{H}_{\|}=P \mathcal{H}$. Projector $P$ can be constructed by means of orthonormal vectors $\left|\psi_{1}\right\rangle,\left|\psi_{2}\right\rangle$,

$$
P=\left|\psi_{1}\right\rangle\left\langle\psi_{1}|+| \psi_{2}\right\rangle\left\langle\psi_{2}\right|
$$

The exact transition operator for $\mathcal{H}_{\|}$is given by the nonzero $(2 \times 2)$ submatrix, $\mathbf{A}(t)$, of the operator $P U(t) P$, where $U(t)$ is the exact transition operator (29) for the 
total state space $\mathcal{H}$ of the system containing the neutral mesons subsystem. So,

$$
\mathbf{A}(t)=\left(\begin{array}{cc}
A_{11}(t) & A_{12}(t) \\
A_{21}(t) & A_{22}(t)
\end{array}\right)
$$

where $A_{j k}(t)=\left\langle\psi_{j}|U(t)| \psi_{k}\right\rangle,(j, k=1,2)$, and $\mathbf{A}(0)=$ $\mathbb{I}_{\|}$. Note that the matrix $\mathbf{A}(t)$ is not unitary. Within the use of this exact transition operator for the subspace $\mathcal{H}_{\|}$ the exact effective Hamiltonian $H_{\|}$governing the time evolution in $\mathcal{H}_{\|}$can be expressed as follows [21-26]

$$
H_{\|}=H_{\|}(t) \equiv i \frac{\partial \mathbf{A}(t)}{\partial t}[\mathbf{A}(t)]^{-1} \text {. }
$$

Thus the exact evolution equation for the subspace $\mathcal{H}_{\|}$has the Schrödinger-like form (36), (7) with timedependent effective Hamiltonian (51),

$$
i \frac{\partial}{\partial t}|\psi, t\rangle_{\|}=H_{\|}(t)|\psi, t\rangle_{\|}
$$

where, $|\psi, t\rangle_{\|}=a_{1}(t)\left|\psi_{1}\right\rangle+a_{2}(t)\left|\psi_{2}\right\rangle=\mathbf{A}(t)|\psi\rangle_{\|} \in$ $\mathcal{H}_{\|}$and $\left.|\psi\rangle_{\|}=a_{1}\left|\psi_{1}+a_{2}\right| \psi_{2}\right\rangle \in \mathcal{H}_{\|}$is the initial state of the system, $\||\psi\rangle_{\|} \|=1$.

It is easy to find from (51) the general formulae for the diagonal matrix elements, $h_{j j}$, as well as for the offdiagonal matrix elements, $h_{j k}$ of the exact $H_{\|}(t)$. We have [25]

$$
\begin{aligned}
& h_{11}(t)=\frac{i}{\operatorname{det} \mathbf{A}(t)}\left(\frac{\partial A_{11}(t)}{\partial t} A_{22}(t)-\frac{\partial A_{12}(t)}{\partial t} A_{21}(t)\right), \\
& h_{22}(t)=\frac{i}{\operatorname{det} \mathbf{A}(t)}\left(-\frac{\partial A_{21}(t)}{\partial t} A_{12}(t)+\frac{\partial A_{22}(t)}{\partial t} A_{11}(t)\right),
\end{aligned}
$$

and so on. Using $(53),(54)$ the difference $\left(h_{11}-h_{22}\right)=2 h_{z}$ playing an important role in relations $(46),(47)$, can be expressed as follows [25]

$$
h_{11}(t)-h_{22}(t)=i \frac{1}{\operatorname{det} \mathbf{A}(t)}\left\{A_{11}(t) A_{22}(t) \frac{\partial}{\partial t} \ln \left(\frac{A_{11}(t)}{A_{22}(t)}\right)+A_{12}(t) A_{21}(t) \frac{\partial}{\partial t} \ln \left(\frac{A_{21}(t)}{A_{12}(t)}\right)\right\} .
$$

Now let us pass to the neutral mesons case, $\left|\psi_{1}\right\rangle \equiv|\mathbf{1}\rangle,\left|\psi_{2}\right\rangle \equiv|\mathbf{2}\rangle$ and let us analyze some consequences of the conservation or violation of $\mathrm{CP}-$, CPT-symmetries in the total system under considerations. If we assume that the system is CPT invariant, that is that (11) holds, then one easily finds that $[11-13,16,25,27]$

$$
A_{11}(t)=A_{22}(t)
$$

Assumption (11) gives no relations between $A_{12}(t)$ and $A_{21}(t)$.

If the system under considerations is assumed to be CP invariant,

$$
[\mathcal{C P}, H]=0
$$

then using the following, most general, phase convention

$$
\mathcal{C P}|\mathbf{1}\rangle=e^{-i \alpha}|\mathbf{2}\rangle, \quad \mathcal{C P}|\mathbf{2}\rangle=e^{+i \alpha}|\mathbf{1}\rangle
$$

one easily finds that for the diagonal matrix elements of the matrix $\mathbf{A}(\mathbf{t})$ relation (56) holds in this case also, and that there is,

$$
A_{12}(t)=e^{2 i \alpha} A_{21}(t),
$$

for the off-diagonal matrix elements.

This means that if the CP symmetry is conserved in the system containing the subsystem of neutral mesons, then for every $t>0$ there must be

$$
\left|\frac{A_{21}(t)}{A_{12}(t)}\right|=1 \equiv \text { const. }
$$

On the other hand, when CP symmetry is violated,

$$
[\mathcal{C P}, H] \neq 0
$$

then one can prove that in such a system the modulus of the ratio $\frac{A_{21}(t)}{A_{12}(t)}$ must be different from 1 for every $t>0$,

$$
[\mathcal{C P}, H] \neq 0 \Rightarrow\left|\frac{A_{21}(t)}{A_{12}(t)}\right| \neq 1, \quad(\forall t>0)
$$

The proof of this property is rigorous (see [26]).

Now let us assume that the CPT symmetry is the exact symmetry of the system under considerations, that is that condition (11) holds. In such a case the relation (56) holds. The consequence of this is that expression (55) becomes simpler and it is easy to prove that the following property must hold [25]

$$
h_{11}(t)-h_{22}(t)=0 \Leftrightarrow \frac{A_{21}(t)}{A_{12}(t)}=\text { const, } \quad(t>0)
$$

Taking into account Khalfin's Theorem, (22), and relations (56), (62) one finds that the following property must hold in the case of the exact effective Hamiltonian for the neutral mesons subsystem: 


\section{K. URBANOWSKI, J. JANKIEWICZ}

\section{Conclusion 2}

If $[\Theta, H]=0$ and $[\mathcal{C P}, H] \neq 0$, that is if $A_{11}(t)=A_{22}(t)$ and $\left|\frac{A_{21}(t)}{A_{12}(t)}\right| \neq 1$ for $t>0$, then there must be $\left(h_{11}(t)-h_{22}(t)\right) \neq 0$ for $t>0$.

So within the exact theory one can say that for real systems, the property (47) cannot occur if CPT symmetry holds and CP is violated. This means that relation (47) can only be considered as an approximation. The question is if such an approximation is sufficiently accurate in order to reflect real properties of neutral meson complexes. One potential solution to this problem is suggested in the next Section, where model calculations are discussed.

\section{MODEL CALCULATIONS}

In this Section we will discuss some results of numerical calculations performed within the use of the Mathematica program for the model considered by Khalfin in $[11,12]$, and by Nowakowski in [16] and then used in $[28,29]$. This model is formulated using the spectral language for the description of $K_{S}, K_{L}$ and $K^{0}, \bar{K}^{0}$, by introducing a hermitian Hamiltonian, $H$, with a continuous spectrum of decay products labeled by $\alpha, \beta$, etc.,

$$
\begin{aligned}
& H\left|\phi_{\alpha}(m)\right\rangle=m\left|\phi_{\alpha}(m)\right\rangle, \\
& \left\langle\phi_{\beta}\left(m^{\prime}\right) \mid \phi_{\alpha}(m)\right\rangle=\delta_{\alpha \beta} \delta\left(m^{\prime}-m\right) .
\end{aligned}
$$

Here $H$ is the total Hamiltonian for the system mentioned in Sections 1, 2 and $4 . H$ includes all interactions and has an absolutely continuous spectrum. We have

$$
\begin{aligned}
\left|K_{S}\right\rangle & =\int_{\text {Spec }(H)} d m \sum_{\alpha} c_{S, \alpha}(m)\left|\phi_{\alpha}(m)\right\rangle, \\
\left|K_{L}\right\rangle & =\int_{\text {Spec }(H)} d m \sum_{\beta} c_{S, \alpha}(m)\left|\phi_{\beta}(m)\right\rangle,
\end{aligned}
$$

and

$$
|\mathbf{j}\rangle=\int_{\operatorname{Spec}(H)} d m \sum_{\alpha} c_{j, \alpha}(m)\left|\phi_{\alpha}(m)\right\rangle,
$$

where $j=1,2$. Thus, the exact $A_{j k}(t)$ can be written as the Fourier transform of the density $\omega_{j k}(m),(j, k=1,2)$,

$$
A_{j k}(t)=\int_{-\infty}^{+\infty} d m e^{-i m t} \omega_{j k}(m)
$$

where

$$
\omega_{j k}(m)=\sum_{\alpha} c_{j, \alpha}^{*}(m) c_{k, \alpha}(m)
$$

The minimal mathematical requirement for $\omega_{j k}(m)$ is the following: $\int_{-\infty}^{+\infty} d m\left|\omega_{j k}(m)\right|<\infty$. Other requirements for $\omega_{j k}(m)$ are determined by basic physical properties of the system. The main property is that the energy (i.e. the spectrum of $H$ ) should be bounded from below, $\operatorname{Spec}(H)=\left[m_{g}, \infty\right)$ and $m_{g}>-\infty$.

Starting from the densities $\omega_{j k}(m)$ one can calculate $A_{j k}(t)$. In order to find these densities from relation (69) one should know the expansion coefficients $c_{j, \alpha}(m)$. Using physical states $\left|K_{S}\right\rangle,\left|K_{L}\right\rangle$ and relations (9), (10) they can be expressed in terms of the expansion coefficients $c_{S, \alpha}(m), c_{S, \alpha}(m)$. Thus, assuming the form of coefficients $c_{S, \alpha}(m), c_{S, \alpha}(m)$ defining physical states of neutral kaons one can compute all $A_{j k}(t),(j, k=1,2)$.

The model considered in $[12,16,28,29]$ is based on the assumption that

$$
c_{S, \beta}(m)=\Theta\left(m-m_{g}\right) \sqrt{\frac{\gamma_{S}}{2 \pi}} \frac{a_{S, \beta}\left(K_{S} \rightarrow \beta\right)}{m-m_{S}+i \frac{\gamma_{S}}{2}},
$$

$$
c_{L, \beta}(m)=\Theta\left(m-m_{g}\right) \sqrt{\frac{\gamma_{L}}{2 \pi}} \frac{a_{L, \beta}\left(K_{L} \rightarrow \beta\right)}{m-m_{L}+i \frac{\gamma_{L}}{2}},
$$

where $a_{S, \beta}$ and $a_{L, \beta}$ are the decay (transition) amplitudes and

$$
\Theta\left(m-m_{g}\right)=\left\{\begin{array}{ll}
1 & \text { if } m \geq m_{g} \\
0 & \text { if } m<m_{g}
\end{array} .\right.
$$

Within this assumption one obtains, for example, that

$$
\mathcal{A}_{S S}(t) \stackrel{\text { def }}{=}\left\langle K_{S}\left|e^{-i t H}\right| K_{S}\right\rangle=\int_{-\infty}^{+\infty} d m \omega_{S S}(m) e^{-i t m}
$$

where

$$
\begin{gathered}
\omega_{S S}(m)=\Theta(m-m g) \frac{\gamma_{S}}{\left(m-m_{S}\right)^{2}+\frac{\gamma_{S}^{2}}{4}} \frac{S}{2 \pi}, \\
S=\sum_{\alpha}\left|a_{S, \alpha}\left(K_{S} \rightarrow \alpha\right)\right|^{2},
\end{gathered}
$$

and so on.

For simplicity, it is assumed in [16] that $m_{g}=0$. So all integrals of the type (72) and (68) are taken between the limits $m=0$ and $m=+\infty$. In [16] all these assumptions made it possible to find analytically amplitudes of type $A_{j k}(t)$ and to express them in terms of the known special functions such as integral exponential functions and the related ones. The same assumptions were used in [28] and will be used in this paper. Note that replacing $\Theta\left(m-m_{g}\right)$ by 1 in (73) leads to a strictly exponential form of amplitudes of the type $\mathcal{A}_{S S}(t)$ as the functions of time $t$. On the other hand, keeping $\Theta\left(m-m_{g}\right)$ results in the presence of additional nonoscillatory terms in amplitudes of the type $\mathcal{A}_{S S}(t), \mathcal{A}_{L L}(t)$ etc. and thus in the amplitudes $A_{j k}(t)$ as well (see $\left.[16,28,29]\right)$.

The results obtained within this model and presented below are obtained assuming that the CPT symmetry holds (i. e. that relations (56) are valid in the model considered) but CP symmetry is violated and by inserting into (71)-(73) and related formulae the following values of the parameters characterizing the neutral 
kaon complex: $m_{S} \simeq m_{L} \simeq m_{\text {average }}=497.648 \mathrm{MeV}$, $\Delta m=m_{L}-m_{S}=3.489 \times 10^{-12} \mathrm{MeV}, \tau_{S}=0.8935 \times$ $10^{-10} \mathrm{~s}, \tau_{L}=5.17 \times 10^{-8} \mathrm{~s}, \gamma_{L}=1.3 \times 10^{-14} \mathrm{MeV}$, $\gamma_{S}=7.4 \times 10^{-12} \mathrm{MeV}$ (see [10]). This model together with the above data makes it possible to examine numerically the Khalfin's Theorem as well as other relations and conclusions obtained using this Theorem (for details see $[16,28,29])$.

The results of numerical calculations of the modulus of the ratio $\frac{A_{12}(t)}{A_{21}(t)}$ for some time interval are presented below in Fig. 1 .

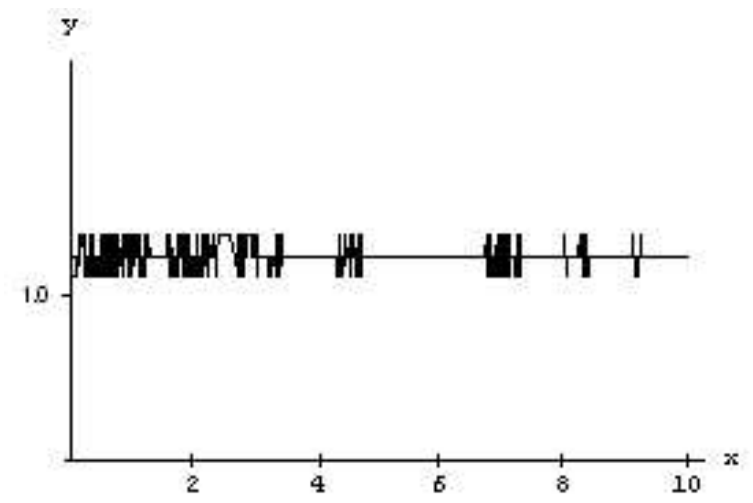

Fig. 1. Numerical examination of Khalfin's Theorem.

Here $y(x)=|r(t)| \equiv\left|\frac{A_{21}(t)}{A_{12}(t)}\right|, x=\frac{\gamma_{L}}{\hbar} \cdot t$, a $x \in(0.01,10)$ and $x, y$ axis are not crossing at the point $(0,0)$.

Analyzing the results of the calculations presented graphically in Fig. 1 one can find using Mathematica that for $x \in(0.01,10)$,

$$
y_{\max }(x)-y_{\min }(x) \simeq 3.3 \times 10^{-16},
$$

where

$$
\begin{aligned}
& y_{\max }(x)=|r(t)|_{\max }, \\
& y_{\text {min }}(x)=|r(t)|_{\text {min }} .
\end{aligned}
$$

So from Fig. 1 and (76) the conclusion follows that if one is able to measure the modulus of the ratio $\frac{A_{12}(t)}{A_{21}(t)}$ only up to the accuracy $10^{-15}$ then one sees this quantity as a constant function of time. The variations in time of $\left|\frac{A_{12}(t)}{A_{21}(t)}\right|$ can be detectable for the experimenter only if the accuracy of his measurements is of the order of $10^{-16}$ or better.

Similarly, using Mathematica and starting from the amplitudes $A_{j k}(t)$ and using relation (55) and condition (56) one can compute the difference $\left(h_{11}(t)-h_{22}(t)\right.$ for the model considered. The results of such calculations for some time interval are presented below in Fig. 2, 3. An expansion of scale in Fig. 2 shows that continuous fast fluctuations, similar to those in Figs. 1 and 3, appear.

There is $y(x)=\Re\left(h_{11}(t)-h_{22}(t)\right.$ and $y(x)=$ $\Im\left(h_{11}(t)-h_{22}(t)\right.$ in Figs 2, 3 respectively. In these Figures $x=\frac{\gamma_{L}}{\hbar} \cdot t, x \in(0.01,5.0)$ and $\Re(z)$ and $\Im(z)$ denote the real and imaginary parts of $z$, respectively. The units on the $y$-axis are in $[\mathrm{MeV}]$.

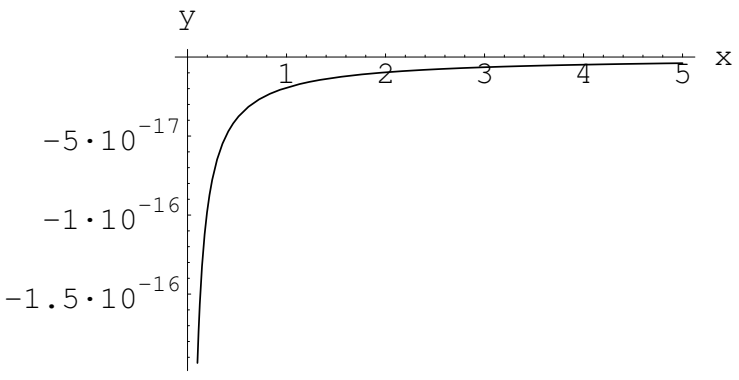

Fig. 2. The real part of $\left(h_{11}(t)-h_{22}(t)\right)$

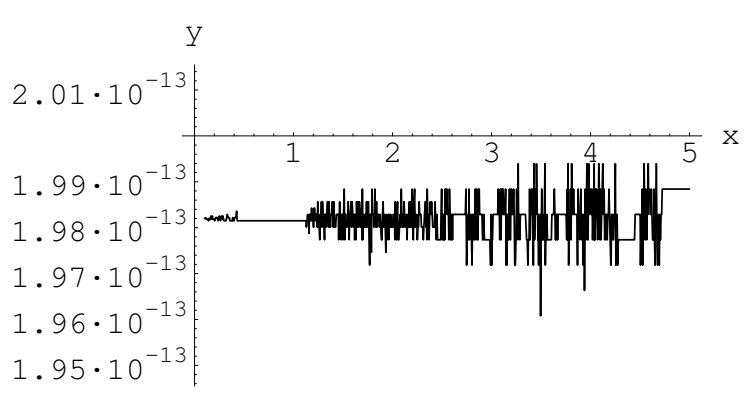

Fig. 3. The imaginary part of $\left(h_{11}(t)-h_{22}(t)\right)$

One can compare the results presented in Figs. 2, 3 with the result obtained analytically. Within the model considered the analytical formulae for the matrix elements $h_{j k}(t),(j, k=1,2)$, were obtained in [28]. Inserting the experimental values of $\tau_{L}, \mu_{L}, \mu_{S}$, etc., mentioned above it is found in [28] for $t=\tau_{L}$ that

$$
\begin{aligned}
& \Re\left(h_{11}\left(t \sim \tau_{L}\right)-h_{22}\left(t \sim \tau_{L}\right)\right) \simeq-4.771 \times 10^{-18} \mathrm{MeV} \\
& \Im\left(h_{11}\left(t \sim \tau_{L}\right)-h_{22}\left(t \sim \tau_{L}\right)\right) \simeq 7.283 \times 10^{-16} \mathrm{MeV}
\end{aligned}
$$

and

$\frac{\left|\Re\left(h_{11}\left(t \sim \tau_{L}\right)-h_{22}\left(t \sim \tau_{L}\right)\right)\right|}{m_{\text {average }}} \equiv \frac{m_{K^{0}}-m_{\overline{K^{0}}}}{m_{\text {average }}} \sim 10^{-21}$,

There is a visible difference between the results presented in Figs. 2, 3 and in (77)-(79). It may be attributed to finite accuracy of numerical calculations performed by Mathematica. No approximations have been used in the analytical calculations.

\section{FINAL REMARKS}

Let us analyze the consequences of the results contained in Sec. II-V for the standard picture of CP violation or possible CPT violation effects in the neutral meson complex. The attention will be focused on the neutral kaon complex as the best studied subsystem of neutral mesons.

Presented in Sec. V are the results of numerical examination of Khalfin's Theorem (see Fig. 1). Note that they 


\section{K. URBANOWSKI, J. JANKIEWICZ}

are in perfect agreement with suppositions formulated in [15] and [26]. One can conclude from these results and from (75) that for the model analyzed and for the times $t$ considered,

$$
\left|\frac{A_{21}(t)}{A_{12}(t)}\right|=R_{0}+\Delta r(t),
$$

where, $R_{0}=$ const, $\Delta r(t)$ varies in time $t$ and $|\Delta r(t)| \leq$ $3,3 \times 10^{-16}$. These results explain why the consequences of Khalfin's Theorem were not yet detected in neutral meson complexes. Simply, the accuracy of tests with neutral mesons was too low.

The consequence of Khalfin's Theorem is Conclusion 2. From this conclusion and from the model calculations performed in Sec. V (see Figs. 2, 3 and results (77)-(79)) one sees that the standard result of the LOY theory, that is the relation (13), is not valid in real systems. This means that this relation (i. e. (13)) is an approximation only.

Another consequence of Khalfin's Theorem is the following one. Namely note that, as it has been proved in Sec. III, the use of the time-independent effective Hamiltonian $H_{\|}$to describe time evolution of the neutral mesons subsystem is inconsistent with the basic assumption of quantum theory that the evolution operator for the total system containing this neutral meson complex must be the unitary operator (see Conclusion 1). This observation means that attempts to describe the neutral meson complexes within the use of any time-independent effective Hamiltonian $H_{\|}$are only approximations. Such attempts may not be able to explain all plausible tiny effects which can be detected in the future more accurate tests with neutral mesons. This also concerns the LOY effective Hamiltonian $H_{\mathrm{LOY}}$. It means that if there exist any effects unexplainable within the LOY approach this need not be signals of "new physics". One should first try to explain such possible effects using more accurate and consistent with the basic assumptions of quantum theory approaches than the LOY method.

In general the form of parameters usually used to describe the scale of CP- and CPT-violation effects depends on the phase used in relations (58) defining the action of $\mathcal{C P}$ operator on the states of neutral $K$ mesons. So, in order to define these parameters it is convenient to choose a phase convention for this operator. For simplicity the following phase convention for neutral kaons is commonly used

$$
\mathcal{C P}|\mathbf{1}\rangle=(-1)|\mathbf{2}\rangle, \quad \mathcal{C} \mathcal{P}|\mathbf{2}\rangle=(-1)|\mathbf{1}\rangle,
$$

instead of the general one (58). Within this phase convention one finds that the vectors

$$
\left|K_{1(2)}\right\rangle \stackrel{\text { def }}{=} \frac{1}{\sqrt{2}}(|\mathbf{1}\rangle-(+)|\mathbf{2}\rangle),
$$

are normalized, orthogonal

$$
\left\langle K_{j} \mid K_{k}\right\rangle=\delta_{j k}, \quad(j, k=1,2),
$$

eigenvectors of the $\mathcal{C P}$ transformation (81),

$$
\mathcal{C P}\left|K_{1(2)}\right\rangle=+(-1)\left|K_{1(2)}\right\rangle
$$

for the eigenvalues +1 and -1 , respectively.

Using these eigenvectors $\left|K_{1(2)}\right\rangle$ of the CPtransformation vectors $\left|K_{L}\right\rangle$ and $\left|K_{S}\right\rangle$ can be expressed as follows $[3,19,30]$

$$
\left|K_{L(S)}\right\rangle \equiv \frac{1}{\sqrt{1+\left|\varepsilon_{l(s)}\right|^{2}}}\left(\left|K_{2(1)}\right\rangle+\varepsilon_{l(s)}\left|K_{1(2)}\right\rangle\right) \text {. }
$$

Within the standard approach the following parameters are used to describe the scale of CP- and possible CPTviolation effects $[3,19,30]$ :

$$
\begin{gathered}
\varepsilon \stackrel{\text { def }}{=} \frac{1}{2}\left(\varepsilon_{s}+\varepsilon_{l}\right) \equiv \frac{h_{12}-h_{21}}{D}, \\
\delta \stackrel{\text { def }}{=} \frac{1}{2}\left(\varepsilon_{s}-\varepsilon_{l}\right) \equiv \frac{h_{11}-h_{22}}{D} \equiv \frac{2 h_{z}}{D},
\end{gathered}
$$

where

$$
D \stackrel{\text { def }}{=} h_{12}+h_{21}+\Delta \mu
$$

and $\Delta \mu=\mu_{S}-\mu_{L}$. According to the standard interpretation following from the LOY approximation, $\varepsilon$ describes violations of CP-symmetry and $\delta$ is considered as a CPT-violating parameter $[3,19,30]$. Such an interpretation of these parameters follows from the properties of LOY theory of time evolution in the subspace of neutral kaons $[2-6,19,27,30]$.

The relations $(85),(86),(87)$ lead to the following formula for the product $\left\langle K_{S} \mid K_{L}\right\rangle$,

$$
\left\langle K_{S} \mid K_{L}\right\rangle \equiv 2 N(\Re \varepsilon-i \Im \delta),
$$

where $N=N^{*}=\left[\left(1+\left|\varepsilon_{s}\right|^{2}\right)\left(1+\left|\varepsilon_{l}\right|^{2}\right)\right]^{-1 / 2}$. Using this relation and the results obtained in Sec. IV and V it is easy to find that $\Im \delta \neq 0$, (see also [31]) in the CPT invariant system. This means that the right hand-side of relation (89) is a complex number and therefore in the case of the conserved CPT- and violated CP-symmetries, in contrast to the standard LOY result, $\left\langle K_{S} \mid K_{L}\right\rangle=|p|^{2}-|q|^{2}$, there must be $\left\langle K_{S} \mid K_{L}\right\rangle \neq\left\langle K_{S} \mid K_{L}\right\rangle^{*}$ in the real systems.

Note that the property $\left\langle K_{S} \mid K_{L}\right\rangle=\left\langle K_{S} \mid K_{L}\right\rangle^{*}$ plays an important role when one applies the Bell-Steinberger unitarity relations [32] for designing or interpreting tests with neutral mesons. So in the light of the above discussion the results obtained in such a way should not be considered as a conclusive evidence, (especially when subtle effects, such as the possible CPT violations, are studied).

From Conclusion 2 from Sec. IV and from the results of the model calculations presented in Sec. V it also follows that the parameter $\delta$ should not be considered as the parameter measuring the scale of possible CPT violation effects: In a more accurate approach [33] and in the exact theory one obtains $\delta \neq 0$ for every system with a violated CP symmetry and this property occurs quite independently of whether this system is the CPT invariant or not. What is more, from Conclusion 2 one finds that if 
CP symmetry is violated and CPT symmetry holds then there must be $\varepsilon_{l} \neq \varepsilon_{s}$ (see (87)) contrary to the standard predictions of the LOY theory. These conclusions are in full agreement with the results obtained in [34] within the quantum field theory analysis of binary systems such as the neutral meson complexes.

It seems that the results following from Khalfin's Theorem and discussed in Sec. III-V have a particular meaning for such attempts to test Quantum Mechanics and the CPT invariance in the neutral kaon complex as those described in [35,36] and recently in [37]. Simply the expected magnitude of the possible effects analyzed in these papers is very close to the results presented in Sec. V and obtained within a more accurate treatment of the neutral kaon subsystem. Another problem with such tests is connected with the results of Sec. III, strictly speak- ing with Conclusion 1. All such tests are planned and interpreted within the theory using a time-independent effective Hamiltonian governing the time evolution in the subspace of neutral kaons $\mathcal{H}_{\|}[35,36]$. From Conclusion 1 one sees that in such a case the evolution operator for the total state space $\mathcal{H} \supset \mathcal{H}_{\|}$cannot be a unitary operator contrary to fundamental assumptions of quantum theory. So it appears that a violation of quantum mechanics is hidden inside the method used to plan and interpret tests detecting such violations. This observation seems to be important because possible CPT or quantum mechanics violation effects are expected to be very tiny. Generally, in the light of the results discussed in Sec. II-V, the interpretation of tests of such tiny effects as a possible CPT violation and a similar one based on the LOY approximation should not be considered as conclusive.
[1] T. D. Lee, R. Oehme, C. N. Yang, Phys. Rev. 106, 340 (1957).

[2] T. D. Lee, C. S. Wu, Ann. Rev. Nucl. Sci. 16, 471 (1966).

[3] J. W. Cronin, Acta Phys. Polon. B 15, 419 (1984); V. V. Barmin et al., Nucl. Phys. B 247, 428 (1984); L. Lavoura, Ann. Phys. (N. Y.) 207, 428 (1991); Buchanan et al., Phys. Rev. D 45, 4088 (1992); C. O. Dib, R. D. Peccei, Phys. Rev. D 46, 2265 (1992); M. Zrałek, Acta Phys. Polon. B 29, 3925 (1998).

[4] in Weak Interaction, edited by M. K. Gaillard, M. Nicolic, Ch. 5, App. A (INPN et de Physique des Particules, Paris, 1977).

[5] T. D. Lee, Particle Physics and Introduction to Field Theory (Harwood academic publishers, London, 1990).

[6] I. I. Bigi, A. I. Sanda, CP Violation (Cambridge University Press, Cambridge, 2001).

[7] G. C. Branco, L. Lavoura, J. P. Silva, CP violation (Oxford University Press, Oxford, 1999).

[8] V. F. Weisskopf, E. P. Wigner, Z. Phys. 63, 54 (1930).

[9] K. Urbanowski, J. Piskorski, Found. Phys. 30, 839 (2000); physics/9803030.

[10] C. Amsler et al., Phys. Lett. B 667, No 1-5, (2008).

[11] L. A. Khalfin, preprints of the CPT, the University of Texas at Austin: DOE-ER-40200-211, February 1990 and DOE-ER-40200-247, February 1991; (unpublished, cited in [13]), and references therein.

[12] L. A. Khalfin, Found. Phys. 27, 1549 (1997).

[13] C. B. Chiu, E. C. G. Sudarshan, Phys. Rev. D 42, 3712 (1990).

[14] P. K. Kabir, A. Pilaftsis, Phys. Rev. A 53, 66 (1996).

[15] P. K. Kabir, A. N. Mitra, Phys. Rev. D 52, 526 (1995).

[16] M. Nowakowski, Int. J. Mod. Phys. A 14, 589 (1999).

[17] G. V. Dass and W. Grimus, Phys. Rev. D 67, 037901 (2003).

[18] G. V. Dass, Phys. Rev. D 60, 017501 (1999).

[19] E. D. Comins, P. H. Bucksbaum, Weak interactions of Leptons and Quarks (Cambridge University Press, 1983); T. P. Cheng, L. F. Li, Gauge Theory of Elementary Par- ticle Physics (Clarendon, Oxford 1984).

[20] K. Urbanowski, Int. J. Mod. Phys. A 7, 6299 (1992).

[21] K. Urbanowski, Bull. de L'Acad. Polon. Sci.: Ser. sci. phys. astron. 27, 155 (1979).

[22] L. P. Horwitz, J. P. Marchand, Helv. Phys. Acta 42, 801 (1969).

[23] K. Urbanowski, Acta Phys. Polon. B 14, 485 (1983).

[24] K. Urbanowski, Phys. Rev. A 50, 2847 (1994).

[25] K. Urbanowski, Phys. Lett. B 540, 89 (2002); hep$\mathrm{ph} / 0201272$.

[26] K. Urbanowski, Acta Phys. Polon. B 37, 1727 (2006).

[27] W. M. Gibson, B. R. Pollard, Symmetry Principles in Elementary Particle Physics (Cambridge University Press, 1976).

[28] J. Jankiewicz, Acta. Phys. Polon. B 36, 1901 (2005).

[29] J. Jankiewicz, Acta. Phys. Polon. B 38, 2471 (2007).

[30] L. Maiani, in The Second DaФne Physics Handbook, Vol. 1, edited by L. Maiani, G. Pancheri, N. Paver, (SISPubblicazioni, INFN-LNF, Frascati, 1995), p. 3.

[31] K. Urbanowski, Mod. Phys. Lett. A 19, 481 (2004).

[32] J. S. Bell, J. Steinberger, in Oxford Int. Conf. on Elementary Particles 19/25 September 1965: Proceedings, edited by T. R. Walsh, A. E. Taylor, R. G. Moorhouse, B. Southworth (Rutheford High Energy Lab., Chilton, Didicot, 1966), p. 195.

[33] J. Jankiewicz, K. Urbanowski, Eur. Phys. J. C 49, 721 (2007).

[34] B. Machet, V. A. Novikov and M. I. Vysotsky, Int. J. Mod. Phys. A 20, 5399 (2005); hep-ph/0407268. V. A. Novikov, hep-ph/0509126.

[35] J. Ellis, J. S. Hagelin, D. V. Nanopoulos, M. Srednicki, Nucl. Phys. B 241, 381 (1984); J. Ellis, N. E. Mavromatos, D. V. Nanopoulos, Phys. Lett. B 293, 142 (1992); J. Ellis, J. L. Lopez, N. E. Mavromatos, D. V. Nanopoulos, Phys. Rev. D 53, 3846 (1996).

[36] P. Huet, M. E. Peskin, Nucl. Phys. B 434, 3 (1995).

[37] J. Bernabeu, J. Ellis, N. E. Mavromatos, D. V. Nanopoulos, J. Papavassiliou, hep-ph/0607322. 
K. URBANOWSKI, J. JANKIEWICZ

\title{
УНІТАРНІСТЬ І ВЛАСТИВОСТІ НЕЙТРАЛЬНОГО МЕЗОННОГО КОМПЛЕКСУ
}

\author{
К. Урбановскі, Ю. Янкєвіч \\ Університет Зельоної Гури, Зельона Гура, Польща, \\ e-mail: K.Urbanowski@proton.if.uz.zgora.pl
}

Проаналізовано доведення теореми Халфіна для нейтрального мезонного комплексу. Показано, що унітарність оператора часової еволюції повної системи підтверджує теорему Халфіна. Обговорено наслідки цієї теореми для системи нейтральних мезонів. Зокрема показано, що діагональні матричні елементи точного ефективного гамільтоніана нейтрального мезонного комплексу не можуть бути однаковими, якщо виконується СРТ-симетрія і СР-симетрія є порушеною. Розглянуто властивості часової еволюції, яку визначає незалежний від часу ефективний гамільтоніан, який діє в підпросторі станів нейтральних мезонів. За допомогою теореми Халфіна показано, що коли такий гамільтоніан не залежить від часу, то оператор еволюції повної системи, яка містить нейтральний мезонний комплекс, не може бути унітарним. Для заданої моделі графічно продемонстровано, як працює теорема Халфіна і показано, як різниця між згаданими діагональними матричними елементами ефективного гамільтоніана змінюється з часом. 